\title{
The Practice of Interventional Gastrointestinal Endoscopy at a Tertiary Referral Hospital
}

\author{
James Waweru ${ }^{1}$, Daniel Ojuka ${ }^{1}$, Pankaj Jani ${ }^{1}$, Wilson Kiraitu ${ }^{2}$, Stephen Onyango ${ }^{2}$, William Okumu ${ }^{2}$. \\ 1. Department of Surgery, University of Nairobi. \\ 2. Endoscopy unit, Kenyatta National Hospital.
}

Correspondence to: Dr. James Waweru, P.O. Box 36153 00200, Nairobi, Kenya. Email: mayshno@gmail.com.

\section{Abstract}

Background: Interventional endoscopy enables one time diagnosis and treatment of gastrointestinal diseases with minimally invasive therapy and improved disease outcome. Local use has remained low with minimal reporting. Knowledge of current practice at a tertiary level may promote utilization of interventional gastrointestinal endoscopy. Objective: To describe the practice of interventional gastro intestinal endoscopy at Kenyatta National Hospital. Methods: In a prospective descriptive study consecutive sampling was used. Main variables were interventional procedure offered, sedation mode and immediate outcome of the procedure. Results: There were 211 cases and interventional endoscopy accounted for $21.7 \%$ of these.

\section{Introduction}

Gastrointestinal system presents a large number of diseases encountered in general practice (1). Gastrointestinal endoscopy is the visual inspection of the digestive canal through a fiber optic cable that utilizes a charge coupled device (2). Early publications on diagnostic endoscopy by Lule and Ogutu et al were with regard to peptic ulcer disease, Helicobacter pylori and gastro-esophageal reflux disease at Kenyatta national hospital in Kenya (3-5). Recently, endoscopic diagnosis of jejuno-gastric intususception has been reported by Mwachiro et al of Tenwek, Kenya (6). Interventional endoscopy allows one time diagnosis and treatment, with added benefits of minimally invasive surgery (7). While innovative technology continuously improve and update diagnosis and treatment of GI diseases in the developed countries, interventional endoscopy has remained at infancy level in developing countries (8). Advances in endoscopic technology and devices have led to a wide variety of new and exciting
The male: female ratio was 1:0.9. The median age group was 41-50 years. Variceal band ligation, ERCP and esophageal stenting were the three most common procedures. No mortalities were recorded during the period of study. Conclusion: Our report describes application of interventional endoscopy in a variety of gastrointestinal diseases with acceptable immediate outcome.

Key words: Interventional, Endoscopy, Varices, Banding

Ann Afr Surg. 2018; 15(1):29-33

DOI:http://dx.doi.org/10.4314/aas.v15i1.7

(C) 2018 Author. This work is licensed under the Creative Commons Attribution 4.0 International License

applications for endoscopy and minimally invasive endoscopic surgical procedures. Variceal hemorrhage is a major cause of upper gastrointestinal bleeding in our local set up. Portal hypertension secondary to schistosomal fibrosis is common in Kenya since schistosomiasis is endemic in both Nyanza and Eastern provinces (9). Sclerotherapy was the first endoscopic modality followed by endoscopic variceal band ligation (EVBL) (10). Lodenyo et al reported successful endoscopic injection sclerotherapy in 112 patients at Kenya Medical Research Institute (KEMRI) in 2007 (11). Jani in 2004 described better results with EVBL regarding variceal eradication time; transfusion requirement and risk of re-bleed (12). Percutaneus Endoscopic Gastrostomy (PEG) is the insertion of a feeding tube into the stomach through the anterior abdominal wall by use of an endoscope. Ponsky and Gauderer first described percutaneous endoscopic placement of gastrostomy tubes in 1980 (13). 
The most common indications for PEG placement are impaired swallowing because of neurological events, oropharyngeal or esophageal tumors, dysphagia, severe facial trauma and poor volitional intake (14). The procedure is cheap, less invasive and no need for general anesthesia in most cases which is a challenging factor in debilitated patients, to whom gastrostomy tubes are most commonly placed (14). Endoscopic ultrasound (EUS) is a technique whereby an ultra sound transducer is incorporated into the tip of the endoscope or a probe is passed through the channel of the endoscope (15). It is now the most accurate imaging technology for staging tumors of the gastrointestinal tract, retroperitoneum and mediastinum (16).

Endoscopic retrograde cholangiopancreatography (ERCP) uses duodenoscopy to identify the major and minor papillae. The biliary and pancreatic ductal systems are cannulated and opacified with contrast material to provide diagnostic information. Other diagnostic tools may be used in conjunction with ERCP including brush cytology, biopsy, intraductal ultrasound, cholangioscopy, and pancreatoscopy (15). Endoscopic gastrointestinal stenting is the use of luminal tubes (Stents) to maintain or restore the lumen of hollow organs (16). Ndonga et al in 2008 reported a series of a hundred endoscopic esophageal stent insertions at St. Mary's hospital in Nairobi for esophageal cancer (17).

Foreign body retrieval from the GI system has been employed using the endoscope. Bane and Bekele (18) have reported their experience of gastrointestinal foreign body extractions under light conscious sedation using flexible video endoscopes in children and adults at Adera Medical center in Addis Ababa, Ethiopia. There is little publication from our local hospital on interventional endoscopy.

\section{Methods}

This was a prospective descriptive study carried out at Kenyatta National Hospital (KNH) endoscopy unit. For a six month period between October 2015 and April 2016, we included patients offered interventional GI endoscopic treatment from twelve years and above. Informed consent was obtained from the patient or the guardian and assent obtained from patients below $18 \mathrm{yrs}$. Consecutive sampling was used. Preformed data sheets were used to fill in collected valuables. Main outcome measures were interventional procedure offered, sedation modalities and immediate outcome of the procedure. The data was stored in a data base using SPSS ${ }^{\circledR}$ for windows v21.0 (Chicago, Illinois). Analysis was done using frequencies and descriptive statistics.

\section{Results}

The number of interventional GI endoscopies was $211(21.7 \%)$ out of the 972 endoscopies done. The median age group was 41-50 years with a range of 13 to 86 years. The male: female ratio was 1:0.9. There were more interventional endoscopies for the upper tract than the lower tract (23.1\% and 5.2\% respectively). Variceal band ligation, ERCP and esophageal stenting were the three common procedures respectively, Figure 1. The least done procedures were argon plasma coagulation, adrenaline injection and endoscopic haemostatic clip application for GI bleeding. Variceal hemostasis was the commonest procedure carried out up to the age of sixty years, after which ERCP and esophageal stenting were commonest. ERCP was twice frequently done in female as compared to males $(M: F=1: 2)$. Distal common bile duct stricture was the commonest indication for ERCP, Table-1. Severe head injury $(n=12)$ was the commonest cause of poor volitional intake requiring PEG fixation. Ten patients had medical co morbidities that included hypertension $(n=7)$, diabetes mellitus $(n=2)$ and deep venous thrombosis $(n=1)$.

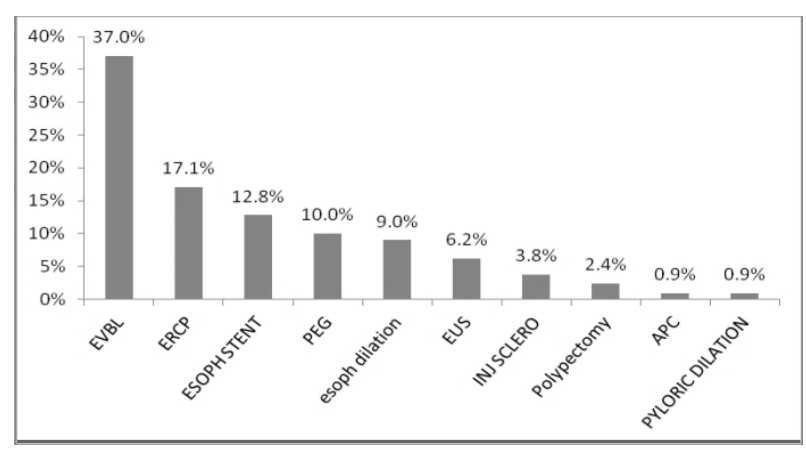

Figure 1: Variety of interventional GI endoscopic procedures

Majority of patients (59.2\%) received two combinations of medications for conscious sedation, midazolam and pethidine (Figure 2). Propofol and ketamine were used during ERCP infrequently as sedatives. Intravenous 
fluids and oxygen via nasal cannula were other supplemental therapies given during the procedures.

Table 1: Causes of obstructive biliary disease

\begin{tabular}{|l|l|l|}
\hline Cause & No & $\%$ \\
\hline Distal CBD stricture & 12 & $35.3 \%$ \\
\hline Cholangiocarcinoma & 8 & $23.5 \%$ \\
\hline Choledocholithiasis & 6 & $17.6 \%$ \\
\hline Pancreatic head tumor & 6 & $17.6 \%$ \\
\hline Peri ampullary tumor & 1 & $3.0 \%$ \\
\hline Primary sclerosingchlangitis & 1 & $3.0 \%$ \\
\hline
\end{tabular}

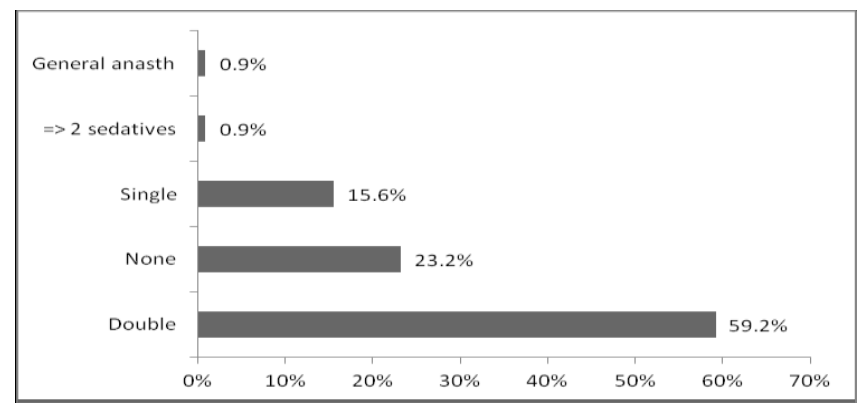

Figure 2: Mode of sedation

Pulse oximetry and pulse rate were used as the sole monitoring variables in $74.9 \%$ of patients, table-2. Blood pressure monitoring was commonly done during ERCP. ERCP had the highest rate of non-completion at $33.3 \%(\mathrm{n}=12)$. Failed ampullary cannulation and pyloric obstruction were among the technical difficulties encountered. Over sedation $(\mathrm{n}=1)$ and continuous bleeding $(n=1)$ were recorded as complications. There were no luminal perforations and no mortalities that were recorded during the period of study.

Table 2: Intra procedural monitoring

\begin{tabular}{|l|l|l|l|l|}
\hline Procedures & None & Sat, P & Sat, P, BP & Sat, P, BP, RR \\
\hline EVBL & $13(16.7$ & $64(82.1 \%$ & $1(1.3 \%)$ & 0 \\
\hline INJ SCLERO & $1(12.5 \%$ & $6(75.0 \%)$ & $1(12.5 \%)$ & 0 \\
\hline ERCP & $1(2.8 \%)$ & $10(27.8 \%$ & $20(55.6 \%$ & $5(13.9 \%)$ \\
\hline Polypectomy & $1(20.0 \%$ & $1(20.0 \%)$ & $1(20.0 \%)$ & $2(40.0 \%)$ \\
\hline Esoph dilation & 0 & $16(84.2 \%$ & $2(10.5 \%)$ & $1(5.3 \%)$ \\
\hline EUS & 0 & $12(92.3 \%$ & 0 & $1(7.7 \%)$ \\
\hline PEG & $2(9.5 \%)$ & $16(76.2 \%$ & $3(14.3 \%)$ & 0 \\
\hline Esoph stent & $1(3.7 \%)$ & $22(81.5 \%$ & $4(14.8 \%)$ & 0 \\
\hline APC & 0 & 0 & $2(100 \%)$ & 0 \\
\hline Pyloric & 0 & $2(100 \%)$ & 0 & 0 \\
\hline
\end{tabular}

P - Pulse, Sat- Saturation, BP- Blood Pressure, RR - Respiratory rate

\section{Discussion}

Our study shows that the commonest indication for interventional GI endoscopy is upper GI bleeding secondary to esophageal or gastric varices. Portal hypertension secondary to schistosomal fibrosis is still common in Kenya complicating into upper GI bleeding (9). Kayamba et al in a study on trends of upper GI endoscopy in Zambia, showed that Esophageal varices were the commonest finding in patients presenting with haematemesis, increasing by $14 \%$ per decade in that patient group (19) Evolution from injection sclerotherapy to endoscopic variceal ligation is portrayed in our findings as well as other studies(12, 20). All patients with esophageal varices underwent variceal band ligation. Injection sclerotherapy was reserved for gastro esophageal junction and fundal varices where banding was impractical. Butyl cyanoacrylate (Histocryl ${ }^{\circledR}$ ) was used as the sclerosant agent which has been shown to achieve variceal hemostasis faster with fewer rebleed as compared to what had been earlier reported by Lodenyo et al with ethanolamine oleate11. Prolonged assisted enteral feeding via nasogastric tube in severe head injured patients is the common indication for percutaneous endoscopic gastrostomy (57.1\%) in our set up. Aggressive management of severe head injury patients in intensive care unit necessitated assisted enteral feeding beyond two weeks. PEG offers superior access to the GI system compared to open surgical methods and is well tolerated with fewer complications (14). All cases of PEG fixation used were done using the pulsion technique as described by Ponsky and Gauderer in 1980 (13). All severe head injury patients brought for PEG fixations were accompanied by anesthesiology resident and two critical care nurses. Ventilation via bag and mask was done through tracheostomy tube and oxygen saturations monitored throughout the procedure. The patients were thereafter wheeled back to ICU and reconnected to the ventilator machine. Other indications for PEG fixation were poor volitional intake due to advanced malignant disease and cerebral vascular accident. ERCP was done as an in-patient procedure. These patients were investigated for hemoglobin level, coagulation tests, liver and renal function tests. Thereafter they were admitted a day before the 
procedure for hydration and six hours fasting. Intravenous ceftriaxone 1 gram was given before the procedure. Obstructive biliary disease was the sole indication for ERCP. The commonest cause of obstructive biliary disease was distal stricture (35.3\%) and cholangiocarcinoma (23.5\%). Adjunct procedures done during ERCP were sphincterotomy of the ampulla, brush cytology of biliary tract, bile stone extraction and biliary stenting. The higher prevalence of cholangiocarcinoma in females may explain the double rate of ERCP in female as compared to male patients. Longer procedure time for ERCP necessitated the use of more potent sedatives, propofol and ketamine. This procedure had also intensive monitoring of blood pressure and respiratory rate in addition to pulse oximetry. ERCP had the highest rate of non-completion at $33.3 \%$ due to failed ampullary cannulation and pyloric obstruction. This may be explained by the long learning curve in ERCP since this procedure began not long ago in our hospital in 2013. Peri ampullary tumors were found to be compressing the duodenum making cannulation of ampulla difficult. Palliative stenting was done for malignant esophageal disease with dysphagia $(n=27)$. Self expandable metallic stents were inserted either by direct endoscopic visualization with scope and stent insitu $(n=8)$ or through metric deployment over guide wire $(n=19)$. Two centimeter overlap of the stent beyond tumor margins was ensured proximally and distally. These patients were thereafter sent for radiotherapy. Esophageal stenting for malignant disease has been shown to effectively palliate inoperable esophageal cancer in resource limited settings $(21,22)$. Bouginage dilation only was done in benign esophageal strictures in 9 patients. Pyloric balloon dilation was carried out in two patients with alkali ingestion however they later underwent surgery for gastric outlet obstruction. Endoscopic ultrasound (EUS) was newly introduced in the facility soon after commencement of this study. EUS was used for diagnosis of pancreatic disease and staging early upper GI tumors. Endoscopic ultrasound guided-fine needle aspiration was carried out in four patients with pancreatic mass. In one patient with chronic abdominal pain due to advanced pancreatic tumor, celiac plexus neurolysis was demonstrated to obliterate afferent pain sensory fibers at the celiac ganglion. Foreign bodies in the esophagus were uncommon above the age of twelve. More so these are largely treated by the ear nose and throat (ENT) specialist in our facility Treated medical co-morbidities (diabetes mellitus, hypertension and deep venous thrombosis) were not a deterrent to interventional endoscopy and did not affect outcome of the procedure. Midazolam and pethidine have been reported to work synergistically for safe sedation and analgesia and were commonly used in this study. Other additional sedatives were used during lengthy procedures like ERCP. Patients who were on sequential EVBL tolerated well the procedure and did not require any sedation. Digital pulse oximeter was affixed to majority of patients during the procedure. Blood pressure monitoring with a brachial cuff was done for complex and lengthy procedures. Indications for oxygen supplementation were elderly age and oxygen saturations less than $90 \%$. No electrocardiographic monitoring was carried out during the procedure. Over sedation and continuous bleeding were recorded as adverse events. Over sedation occurred in patients who received multiple doses of two or more sedatives and were managed by oxygen supplementation and monitoring by a recovery nurse until fully awake. One patient had continuous bleeding from duodenal ulcer. Argon plasma coagulation and adrenaline injection were employed with hemostasis. Later in the ward the patient had continued hematemesis and was taken to theatre for laparotomy where the bleeding vessel was over sewn. There were no luminal perforations and no mortalities that were recorded during the period of study. With adequate skill and preparation, interventional endoscopy has minimal complications that can be managed with good outcome.

\section{Conclusion}

Our report describes application of interventional endoscopy in a variety of gastrointestinal diseases with acceptable immediate outcome. This may increase awareness among clinicians, promote more training and advocacy in health policy with the sole aim of improving standards of gastroenterology healthcare in our set up. 


\section{References}

1. Makmun D. Present Status of Endoscopy, Therapeutic Endoscopy and the Endoscopy Training System in Indonesia. Gastrointest. Endosc. 2014; 26 (52):2-9.

2. Hamilton B, McNeil L. Short Practice of surgery 25th edition chapter 11 pg $151-66$.

3. Lule GN, Wankya B.M, Shah MV et al. Peptic Ulcer Disease at Kenyatta National Hospital: An Endoscopic Experience. East Afr Med J. 1987; 64(10) 638-42.

4. Karari EM, Lule GN, Mc Ligeyo SO, et al. Endoscopic Findings and the Prevalence of Helicobacter Pylori in Chronic Renal Failure Patients with Dyspepsia. East Afr Med J. 2002; 77(8):406-9.

5. Maende JA, Ogutu EO, Nyong'o A, et al. Upper Gastrointestinal Mucosal Lesions in Dyspeptic Patients with Homozygous Sickle Cell Disease in Kenya. East Afr Med J. 1998; 75 (3) 148-50.

6. Mwachiro MM, Burget SL, Arega FL et al. Endoscopic Diagnosis of Jejuno-gastric intususception. Ann. Afr. Surg.2014; 12 (1):39-41.

7. Lindsay JO, Andreyev HJ, Vlavianos P, et al. Selfexpanding Metal Stents for the Palliation of Malignant Gastroduodenal Obstruction in Patients Unsuitable for Surgical Bypass. Aliment Pharmacol Ther. 2004; 19 (8):901-5.

8. Watermeyer G, Van Wyk ME, Goldberg PA et al. Audit of Provincial Gastroenterology Services in the Western Cape. S Afr J Surg. 2008; 46(3):68-72.

9. Lule GN, Obiero ET, Ogutu EO. Factors that Influence the Short-term Outcome of Upper Gastrointestinal Bleeding at Kenyatta National Hospital. East Afr Med J. 1994; 71:240-5.

10. Jani PG. Oesophageal Variceal Banding: Report of the First Eight Cases in Kenya. East Afr Med J. 1997; 74: 395-6.

11. Lodenyo H and Okoth FA. An Observational Study on Oesophageal Variceal Endoscopic Injection Sclerotherapy in Patients with Portal Hypertension seen at the Centre for Clinical Research, Kenya Medical Research Institute. Afr J Health Sci. 2007; 14:216-8.
12. P.G Jani. Endoscopic Variceal Band ligation: A Local Experience. East Afr Med J. 2004; 81(4):2124.

13. ASGE technology committee. Endoscopy. Role of Endoscopy in Enteral Feeding. Gastrointest Endosc.2002; 55:794-7.

14. Rahnemai-Azar A, Rahnemaiazar A, Naghshizadian $\mathrm{R}$ et al. Percutaneous Endoscopic Gastrostomy: Indications, Technique, Complications and Management. World J Gastroenterol. 2014; 20(24): 7739-51.

15. Varadarajulu S, Banerjee S, Barth BA, et al. GI endoscopes. Gastrointestinal Endoscopy. 2011; 74(3):1-6.e.

16. ASGE technology committee. Technology Status Evaluation Report on Enteral Stents. Gastrointest Endosc. 2011; 74(3 :) 455-64.

17. Ndonga AK, Rucha M, Oigara R. Oesophageal Cancer and Experience with Endoscopic Stent intubation at St. Mary's Hospital, Nairobi. Ann Afr Surg. 2008; 3:21-6.

18. Bane A, Bekele A. Management of Gastrointestinal Foreign bodies using Flexible Endoscopy: An experience from Addis Ababa, Ethiopia. East Cent Afr J Surg. 2012; 17(3) $32-8$.

19. Kayamba V, Sinkala E, Mwanamakondo S et al. Trends in Upper Gastrointestinal Giagnosis Over Four Decades in Lusaka, Zambia: A Retrospective Analysis of Endoscopic Findings. BMC Gastroenterol.2015; 6(15):127.

20. Alatise OI, Aderibigbe AS, Adisa AO, et al. Management of Overt Upper Gastrointestinal Bleeding in a Low Resource Retting: A Real World report from Nigeria. BMC Gastroenterol. 2014; 10(14):210.

21. Russell E.W, Robert K.P, John W.F et al. Stents as Sole Therapy for Oesophageal Cancer: A Prospective Analysis of Outcomes after Placement. Lancet Oncology: 2009; 10(3):240-6.

22. White RE, Chepkwony R, Mwachiro M et al. Randomized Trial of Small-diameter Versus Largediameter Esophageal Stents for Palliation of Malignant Esophageal Obstruction. J Clin Gastroenterol. 2015;49(8):660-5 